\title{
Social Resilience: The Forgotten Dimension of Disaster
}

\section{Risk Reduction}

\author{
Guy Sapirstein
}

\section{Background}

The current thinking in the Disaster Risk Reduction field emphasizes assessment and reduction of vulnerability and especially social vulnerability as an important factor in mitigating the effects of disasters. In the process of emphasizing vulnerability, the role and complexity of social resilience was somewhat lost and at times minimized. For example, Terry Cannon and his colleagues include resilience as a factor of social vulnerability in a report to United Kingdom Department for International Development (DFID) (Cannon, Twigg and Rowell, 2002). The United Nations University, Institute for Environment and Human Security (UNU-EHS) delineates "Social Vulnerability" and "Individual Vulnerability" as working areas, but does not mention Social or Individual Resilience (Bogardi, 2006).

As a result of the conflation of the term 'social resilience' with 'social vulnerability', these two concepts in addition to the concept of "capacity" became somewhat blurred. Cannon et al. (2002) describes some of the confusion around the concepts of social vulnerability and capacity in terms of their relationship to one another. At times they appear linearly related and at other times nonlinearly related. Jeff DaytonJohnson (2004) clarifies this confusion by explaining that "Adaptive capacity is a function of countries ex-ante vulnerability to natural disaster risk and their ex-post resilience once such disasters have struck “ (p. 8). In other words, both vulnerability and resilience affect capacity.

\section{The relationship between Social Vulnerability and Social Resilience}

The concepts of social vulnerability and social resilience are orthogonal to each other (Bogardi, 2006). In other words, when represented on a graph, social vulnerability and social resilience are perpendicular to one another (one is represented on the " $X$ " axis and the other represented on the " $Y$ " axis). Figure 1 shows a schematic depiction of social resilience and social vulnerability. ${ }^{1}$ 
Graph 1: Schematic depiction of the relationship between Social Resilience and Social Vulnerability

Time

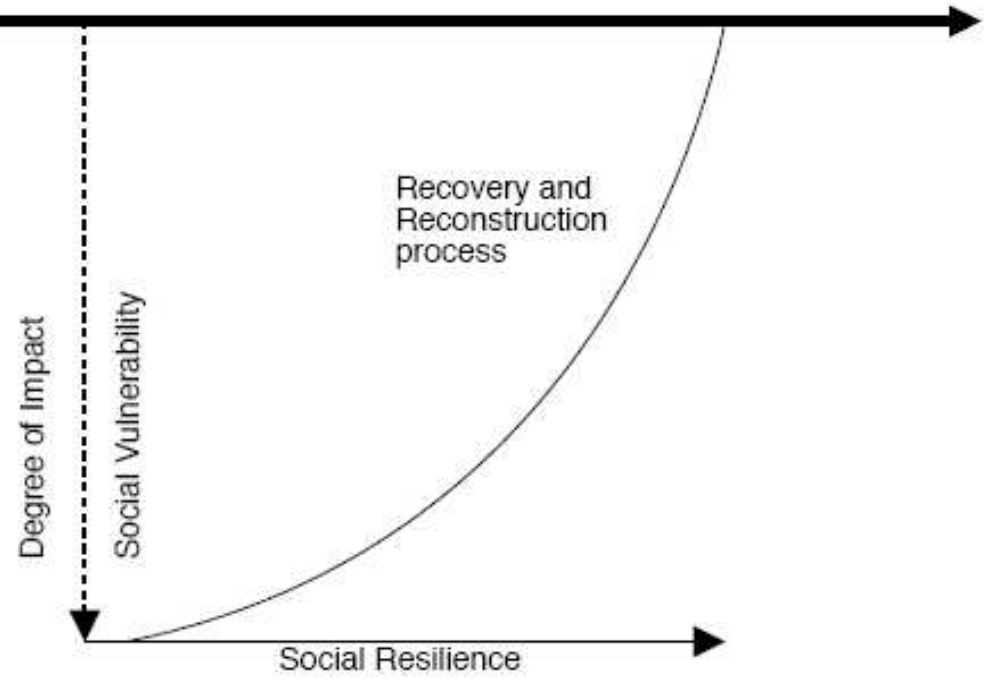

Figure1: Schematic depiction of social resilience and social vulnerability

While they might interact with respect to their effect on community capacity, they are not directly correlated with each other. Moreover, they are measured in different units. Vulnerability is the degree to which people will be impacted by a hazard (natural or man made). Social vulnerability is measured primarily through cost: be it economic or human. In other words, how much will it cost if a hazard occurs in a given community and how many lives will be lost or affected. Social resilience, in contrast, is measured by time. Specifically - how long would it take for the community to respond to the event, self organize and incorporate the lessons learned before returning to a [new] normal way of functioning. While it might be tempting for some to transform this measure of "time" to economic cost (time=money), that would effectively force a nonlinear relationship into a linear one. The amount of time it takes to recover from an occurrence of a hazard affects not only the economic viability of a community, but also its social fabric or "glue" that keeps it together. 
The longer it takes to recover, the more likely it is that the community will break up because of people leaving, economic stagnation, and rampant psychological and emotional distress. Similar to the notion of "broken families", the effect of psychological or relationship dysfunction reaches beyond the economic impact of that lack of functioning.

A concept analogous to the concept of social resilience is the concept of Recovery Time Objectives (RTOs) which is taken from the field of Business Continuity Planning $(\mathrm{BCP})$. It refers to the time it should take a company (or unit) to resume operations after a critical disruption. The purpose of this metric is to create a benchmark measure that can help evaluate the efficiency and capacity of a business unit to cope with a critical event or process.

Emphasizing the importance of time in the recovery from disasters is not merely a cold hearted corporate attitude that time=money. It is also a reflection of the reality that the longer it takes to move from response to rebuilding and reconstruction, the more deleterious and long lasting the negative effects of the disaster will be. In the field of human trauma it is known that while people need some amount of time to recuperate from traumatic events, they should try to resume their regular or necessary activities as soon as possible.

\section{Definition of Resilience}

Despite outlining a clear description of the relationship between resilience, vulnerability and capacity, Dayton-Johnson (2004) does not clearly define resilience beyond its economic aspect. In fact, few (if any) of the people who have written on social vulnerability have defined social resilience clearly. The abundance of definitions for "resilience" and the fact that this concept is shared by many different disciplines make it particularly difficult to define uniformly. Two definitions that are especially comprehensive are the following:

- ..."the ability to face internal or external crisis and not only effectively resolve it but also learn from it, be strengthened by it and emerge transformed by it, both individually and as a group." (Brenson-Lazan, 2003). 
- "The resilience of an eco-system is its capacity of absorb disturbances while maintaining its behavioral processes and structure. It can be defined as the capacity to buffer perturbations, to self-organize, and to learn and adapt." (ResAlliance.org).

These definitions include 4 main components: Response, Self-Organization, Learning, and Adaptation².

\section{Response}

The social response to hazards (natural or man-made) differs across regions and cultures. Communities differ with respect to their reliance on themselves versus others in the aftermath of a hazardous event. More resilient communities mobilize their own resources quickly and effectively, irrespective of assistance from other sources. Often it is community inaction (passively waiting for assistance) that contributes greatly to psycho-social complications following hazardous events (Hutton, 2001).

\section{Self Organization}

From a social perspective, self organization refers to the activities a community engages in to restore functioning at least to the pre-event level. These activities can range from emergency response and recovery to fostering collaboration among civic organizations and neighborhoods and maintaining social order. Community initiative is important in providing a sense of safety, control of one's destiny, and predictability, following a profound disruption of all three (Herman, 1992).

\section{Learning}

Coming together as a community to discuss "lessons learned" after negative (and positive) events have taken place is sometimes a challenge (Bazerman and Watkins, 2004). People often want to "move on" and deal with their current stressors. When disasters are seen as "an act of god" or "fate" rather than a preventable phenomenon learning is tacitly discouraged. Combining the process of community learning with educational curricula is an especially important step, as it empowers children to respond responsibly to natural hazards and fosters a culture of learning. 


\section{Adaptation}

Catastrophic events cause change. Very often, communities are forever changed either through loss of life or loss of social-cultural landmarks and reference points. While grief is understandable and normal, it is important for a community to adapt to the new normal. The process of adaptation ensures that people are dealing with the situation at hand, rather than romanticizing an idealized past or harboring anger and resentment at perceived (or real) failures of government.

These components apply equally to all social units - from individuals, through families to communities or organizations. Moreover, they necessitate voluntary participation by all involved: one cannot force or require an individual to be resilient. Rather, resilience is the outcome of developing those four components. While poverty reduction (the cornerstone of reducing social vulnerability) is a complex process involving factors beyond the individual or even a community, developing social resilience is a grassroots process that does not require an intervention on a macro level. Social resilience can be achieved at the level of the individual family, isolated village or at the other end of the spectrum - a mega-city.

\section{Redundancy: Enhancing Social Resilience}

Implicit in the definitions of resilience is the assumption of redundancy (ResAlliance.org). In other words, there is more than one system of coping - when one system is impacted the other systems help with continued functioning. The greater the redundancy, the more resilient the system. A simple example would be someone who has a single computer with no backup. If the computer crashes they will have lost all the data. Increasing redundancy would translate into having a backup and perhaps even having another computer with the data on it (higher level of redundancy). Looking at the amount of time it would take that person to resume working (resilience), one could safely assume that if they had a backup and an extra computer the disruption would be minimal. If they only had the backup they would need to find another computer which would take longer (thereby decreasing resilience). 
In the social sphere, social resilience assumes a certain amount of redundancy. Traditionally, the redundancy was created through family ties. People were cared for by their extended family when in need (e.g. disaster, illness, old age). With the phenomenon of migration to more urban areas, that traditional system of social resilience cannot always provide support (e.g. Klineberg, 2002). In these situations the creation of redundancy is expected of the central or local government which is supposed to take care of the citizens and residents when they cannot care for themselves (e.g. Vatsa 2005).

With globalization, population migration and other social phenomena of the late $20^{\text {th }}$ century the Disaster Risk Reduction community needs to rethink the way in which redundancy and social resilience are achieved. An increasing number of people are living in urban areas -- at least $50 \%$ of the world population by some estimates (Vatsa, 2005) -- and thus are exposed to different types of social ties. Faith-based communities, workplace communities, neighborhoods, dwellers of high-rise buildings all can provide the redundancy necessary for social resilience. Of this list, the first two have a particular vested interest in social resilience. Most (if not all) organized religions have as part of their tenets a moral code calling for social responsibility (www.wcrp.org under 'initatives'. See also Brammer, Williams and Zinkin, 2005 for an interesting perspective). Similarly, most (if not all) companies and businesses depend on people for their functioning and revenue. It seems logical then that those organizations (faith based and businesses), should be involved in the process of enhancing social resilience. Not just out of a sense of social responsibility, but out of a realization that social resilience is critical and essential to their continued viability. A business will cease to function if it does not have employees, customers or suppliers. Even if it has all three, the longer the disruption to operations, the more money the business will lose. Similarly, a faith based community will fail its constituents if it does not offer social support. With many more alternatives in an urban setting, worshipers are more likely to choose a community offering them organized, practical and sustained support in times of crisis and disaster.

An important aspect of this approach is the adoption of a decentralized component to enhancing social resilience - much of this process is a local, lateral approach, rather than the traditional top-down approach. In this respect it differs from many of the policies aimed at reducing social vulnerability (e.g. DFID, 2006; Twigg, 2005; Vatsa, 2005). 
Many such programs are dependent on central governance to assist in attaining the goals. Social resilience is developed community by community. Assistance from centralized or local government is of secondary importance. As long as there is buyin from organizations in the community - employers, businesses, faith-based organizations, community groups, etc. -- one can efficiently and cost-effectively enhance the ability of communities to "bounce forward" after a potentially devastating event.

Introducing a greater degree of redundancy into prevention and mitigation of the impact of hazards reduces the pool of people who are dependent solely on governments and charitable organizations for care, thereby facilitating a greater efficiency in response to hazards. This efficiency is the cornerstone to the enhancement of social resilience. With more "safety nets" two goals will be accomplished:

1. Governments and charitable organizations will be able to attend to the most needy.

2. Less people will "slip through the cracks" and be missed in the planning, response and recovery phases.

\section{Future Steps to Enhancing Social Resilience}

The biggest obstacle to implementing a plan for enhancing social resilience is obtaining the cooperation and collaboration of all the "players" or stakeholders who together create the matrix of redundancy. The challenge to the business or private sector is to look beyond the immediate profit and loss statements and invest in strategic planning that will ultimately enhance social resilience. In fact there is research that suggests that some types of corporate support is useful in reducing absenteeism and worker's compensation claims (Sanchez, Korbin and Viscarra, 1995). The public sector needs to foster a collaborative atmosphere and consider incentivizing the other players to assume some of the responsibility for enhancing the social resilience. NGO's and other traditional "not-for-profit" entities need to accept the private -- "for profit" -- sector as having an important role in this process, rather than being suspicious and critical of its motives. 
The nascent field of Human Impact Preparedness provides insight into strategies that would be useful to employ in addressing social resilience. Human Impact Preparedness addresses disasters and their mitigation from a purely human lens, rather than the other lenses (technology, infrastructure, communication, etc.) which address people only indirectly.

\section{Conclusion}

Increasing the strength of a society is about increasing the strength and scope of the internal connections between the people, organizations and environment that form that society. Moving away from the doctrine of independence to embracing a culture of interdependence is the key to both harmony and development. The essence of social resilience is not merely fostering independence, but rather the interdependence that is known in the Philippines as "kapwa" (the unity of "self" and "other") and in South Africa as "ubuntu" (the spirit of a community) or "Umuntu ngumuntu ngbaantu" (I am a person through other people). The cultural knowledge of social resilience exists across the globe, but it is up to policy makers, the private sector, and communities to apply it. 

${ }^{1}$ The author acknowledges that the process of recovery is far more complex than appears in
this representation which is solely for the purpose of representing social resilience and social
vulnerability. Zunin and Meyers (2000) provide an in depth description of the process.
2 As concept of resilience is shared by many different fields, the term resilience as used in
this article refers to 'the general capacity to recover'. Social resilience refers to the application
of this term specifically to the social aspect of resilience. 


\section{References}

BAZERMAN, M. H., AND WATKINS, M. D. (2004). Predictable Surprises. Boston, MA: Harvard Business School Press.

BOGARDI, J. J. (2006). Resilience building: from knowledge to action. Introduction to UNU-EHS . June, Presented to the Summer Academy of UNU:EHS. [available online] http://www.ehs.unu.edu/file.php?id=184

BRENSON-LAZAN, G. (2003). Group and social resilience Building. [available online] www.communityatwork.com/resilience/RESILIENCIAENG.pdf

CANNON, T., TWIGG, J., AND ROWELL, J. (2002). Social Vulnerability, Sustainable Livelihoods and Disasters: Report to DFID Conflict and Humanitarian Assistance Department (CHAD) and Sustainable Livelihoods Support Office. [available online] http://www.livelihoods.org/info/docs/vulnerability.doc

DAYTON-JOHNSON, J. (2004). Natural Disasters and Adaptive Capacity. OECD Development Centre, working paper No. 237.

DFID (2006). Reducing the Risk of Disasters - Helping to Achieve Sustainable Poverty Reduction in a Vulnerable World: A DFID policy paper. [available online] http://www.unisdr.org/news/DFID-reducing-risk-of-disasters.pdf.

HERMAN, J. L. (1992). Trauma and Recovery. Boston, MA: Basic Books.

HUTTON, D. (2001). Psychosocial Aspects of Disaster: Integrating Communities Into Disaster Planning and Policy Making. Institute for Catastrophic Loss Reduction. [available online] www.ICLR.org. 
KLINEBERG, E. (2002). Heat Wave: A Social Autopsy of disaster in Chicago. Chicago, IL: University of Chicago Press.

SANCHEZ, J. I., KORBIN, W. I., AND VISCARRA, D. M. (1995). Corporate Support in the Aftermath of a Natural Disaster: Effects on Employee Strains. Academy Of Management Journal, 38(2), 504-521.

RESILIENCE ALLIANCE, http://www.ResAlliance.org/576.php Date of access September 28, 2006.

TWIGG, J. (2005). Community Participation: Time for a Reality Check? (in Jeggle, T., ed. Know Risk. UN/ISDR: Tudor-Rose).

VASTA, K. S. (2005). Home for Almost Half the World. (in Jeggle, T., ed. Know Risk. UN/ISDR: Tudor-Rose).

WORLD CONFERENCE OF RELIGIONS FOR PEACE, http://www.wcrp.org/about/index, Dat of access October 23, 2006.

ZUNIN, L. M. \& MYERS, D. (2000). Training Manual for Human Service Workers in Major Disasters. $2^{\text {nd }}$ Ed. Washington, DC: Department of Health and Human Services Substance Abuse and Mental Health Services Administration, Center for Mental Health Services; DHHS Publication No. ADM 90-538. [available online] http://www.mentalhealth.org/publications/allpubs/ADM90-538/tmpreface.asp Datw of access June 14, 2005 